\title{
A Turning Strategy of a Multi-Legged Locomotion Robot
}

\author{
Kazuo Tsuchiya, Shinya Aoi, Katsuyoshi Tsujita \\ Dept. of Aeronautics and Astronautics, Graduate School of Engineering, Kyoto University \\ Yoshida-honmachi, Sakyo-ku, Kyoto, 606-8501, Japan, shinya_aoi@kuaero.kyoto-u.ac.jp
}

\begin{abstract}
In this paper, first, stability of locomotion of the multilegged locomotion robot along a straight line is analyzed, and then, based on the dynamic characteristics, a turning strategy is proposed. The effectiveness of the turning strategy is verified by the numerical simulations; the robot can turn with less slip to the ground by decreasing the stability of the straight walk of the robot.
\end{abstract}

\section{Introduction}

Locomotion with legs allows a robot to move on a rough terrain. Moreover, locomotion with many legs has the advantage to improve the stability because the robot is supported with many legs. But it also has the drawback to lack the maneuverability of locomotion because the robot is constrained on the ground by many legs. From the practical view point, it is important to study how to improve the maneuverability of a multilegged locomotion robot. Now the established method to control a robot consists of motion planning and motion control. Motion planning is to compute the motions of the joints so as to realize a given motion of the robot, and motion control is to control the joints so as to realize the designed motion. Motion planning of the turning walk of a multi-legged locomotion robot is to compute the positions of the tips of the legs so that the robot moves along a given curved line, and this results in the inverse problems of the equations of motion for many variables. This calculation is not efficient, and the solutions can not adapt to the change of the environment.

In ethology, research on arthropod locomotion is progressed. Arthropod locomotion has much stability and also maneuverability such as quick turning. Now, research is focused to reveal what mechanism of arthropod can make the locomotion stable and maneuverable[1]. Schmitt[2], et al. have analyzed the dynamics of the turning behavior of the cockroach Blaberus discoidalis based on a mathematical model and revealed that the cockroach decreases the stability of a straight walk by moving the points on the body where the forces from the ground act and this, in turn, can make the cockroach turn quickly.

In the preceding researches on robotics, there is little research of locomotion to enhance the maneuverability of motion of a robot by changing the dynamic characteristics of the robot. This paper deals with turning strategy of a multi-legged locomotion robot. We propose the control strategy for the robot so as to realize to turn efficiently by decreasing the stability of the straight walk of the robot. We have already proposed the locomotion control system of a multi-legged locomotion robot using oscillators. In this paper, the robot with this control system is subject of investigation. First, we analyze the stability of the straight walk of the robot with the proposed control system. The analysis reveals that by changing a certain control parameter, the stability of the straight walk of the robot decreases and beyond a critical point the meandering motion appears. We develop the control strategy for the multi-legged locomotion robot to turn efficiently using the change of the dynamic characteristics of the robot.

\section{3-D dynamics model}

We consider the multi-legged locomotion robot shown in Fig.1. The robot has six modules, each of which has one body and two legs. Each leg is composed of two links which are connected to each other through a one degree of freedom rotational joint and are connected to the body through a one degree of freedom rotational joint. Each module is connected to each other through a coupler which composed of roll, pitch and yaw joints. The inertial fixed coordinate system is defined as $\left[\boldsymbol{a}_{0}\right]=\left[\boldsymbol{a}_{01}, \boldsymbol{a}_{02}, \boldsymbol{a}_{03}\right]$. The coordinate system fixed on the body of first module is defined as $\left[\boldsymbol{a}_{1}\right]=\left[\boldsymbol{a}_{11}, \boldsymbol{a}_{12}, \boldsymbol{a}_{13}\right]$. Axes 1,2 and 3 coincide with the direction of roll, pitch and yaw, respectively. The modules are enumerated from 1 to 6 and the coupler between module $(i-1)$ and module $i$ is numbered as coupler $i$, as shown in Fig.1. Left and right legs are numbered as leg 1 and leg 2, respectively. The joints 
and the links of each leg are numbered as joint 1 and 2 , and link 1 and 2 as shown in the figure. The position vector from the origin of $\left[\boldsymbol{a}_{0}\right]$ to the origin $\left[\boldsymbol{a}_{1}\right]$ is defined as $\boldsymbol{r}_{0}=\left[\boldsymbol{a}_{0}\right] r_{0}$. We define $\theta_{i}$ as the components of 3-1-2 Euler angle from $\left[\boldsymbol{a}_{i-1}\right]$ to $\left[\boldsymbol{a}_{i}\right]$, and define $\theta_{i k}^{(j)}$ as the joint angle of link $k$ of leg $j$ of module $i$.

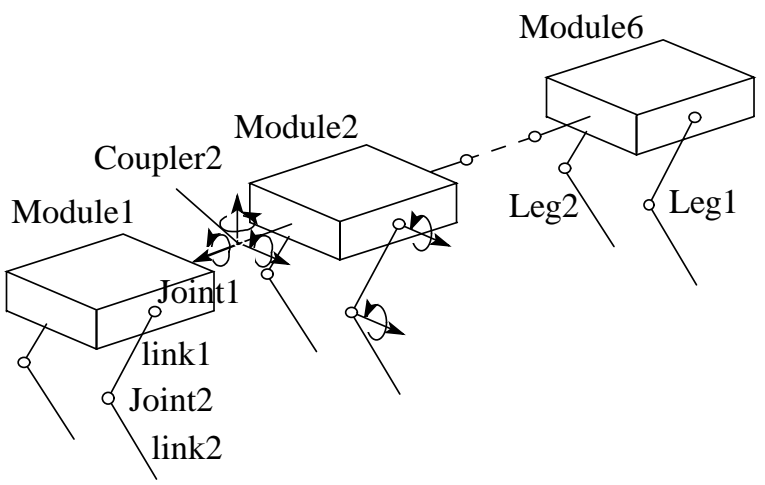

Figure 1: Schematic model of multi-legged locomotion robot

The state variables are defined as follows;

$$
\begin{aligned}
& q^{T}=\left[r_{0 m} \theta_{i m} \theta_{i k m}^{(j)}\right] \\
& i=1, \cdots, 6, j=1,2 \\
& k=1,2, m=1,2,3
\end{aligned}
$$

Equations of motion for state variable $q$ are derived using Lagrangian formulation as follows;

$$
M \ddot{q}+H(q, \dot{q})=G+\sum\left(\tau_{i}+\tau_{i k}^{(j)}\right)+\Lambda
$$

where $M$ is the generalized mass matrix. $H(q, \dot{q})$ is the nonlinear term which includes Coriolis forces and centrifugal forces. $G$ is the gravity term. $\tau_{i}$ and $\tau_{i k}^{(j)}$ are the input torques of the actuators at coupler $i$ and joint $k$ of leg $j$ of module $i$. $\Lambda$ is the reaction force from the ground where the tip of the leg makes contact. The reaction forces are modeled using a spring and damper model.

\section{Control system of locomotion[3]}

The control system of locomotion is composed of leg motion controllers and a gait pattern controller(Fig.2). The leg motion controllers drive the joint actuators of the legs so as to realize the desired motions commanded by the gait pattern controller. The gait pattern controller involves nonlinear oscillators corresponding to each leg. It receives the commanded signal of the nominal gait pattern as the reference, and generates a gait pattern which is served to the leg motion controller as the commanded signal.

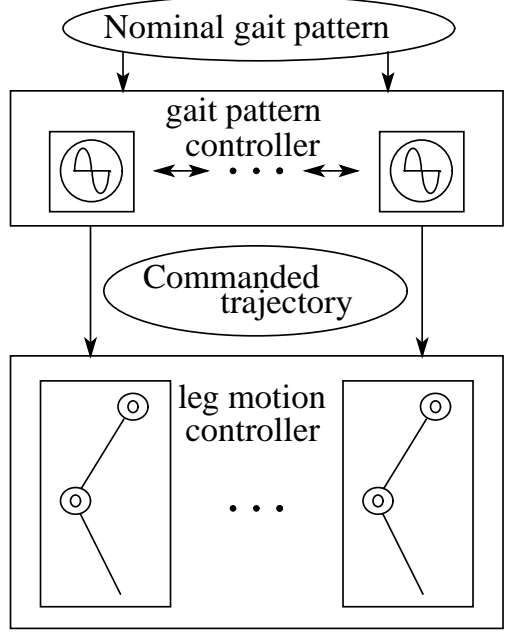

Figure 2: Control system of locomotion

The nominal trajectories of the tips of the legs are designed as follows; First, we define the position of the tip of the leg where the transition from the swinging stage to the supporting stage as the anterior extreme position (AEP) and the position where the transition from supporting stage to the swinging stage as the posterior extreme position (PEP). The nominal PEP and the nominal AEP are expressed as $\hat{\eta}_{i P}^{(j)}, \hat{\eta}_{i A}^{(j)}$ in the coordinate system $\left[\boldsymbol{a}_{i}\right]$. The nominal trajectory for the swinging stage is designed as a closed curve $\hat{\eta}_{i S w}^{(j)}$ which involves the points $\hat{\eta}_{i A}^{(j)}$ and $\hat{\eta}_{i P}^{(j)}$, and the nominal trajectory for the supporting stage is designed as a straight line $\hat{\eta}_{i S p}^{(j)}$ which also involves the points $\hat{\eta}_{i A}^{(j)}$ and $\hat{\eta}_{i P}^{(j)}$. Then, these trajectories are given as functions of the phases of the oscillators. The state of the oscillator $(i, j)$, oscillator on leg $j$ of module $i$, is expressed as follows;

$$
z_{i}^{(j)}=\exp \left(\boldsymbol{j} \phi_{i}^{(j)}\right)
$$

where $z_{i}^{(j)}$ is the state of the oscillator and $\phi_{i}^{(j)}$ is the phase of the oscillator. we set the nominal phase dynamics of the oscillator as

$$
\dot{\hat{\phi}}_{i}^{(j)}=\hat{\omega}
$$

The nominal trajectories $\hat{\eta}_{i S w}^{(j)}$ and $\hat{\eta}_{i S p}^{(j)}$ are given as functions of the nominal phase $\hat{\phi}_{i}^{(j)}$ of the oscillator as

$$
\begin{aligned}
\hat{\eta}_{i S w}^{(j)} & =\hat{\eta}_{i S w}^{(j)}\left(\hat{\phi}_{i}^{(j)}\right) \\
\hat{\eta}_{i S p}^{(j)} & =\hat{\eta}_{i S p}^{(j)}\left(\hat{\phi}_{i}^{(j)}\right)
\end{aligned}
$$

where the nominal phases $\hat{\phi}_{i}^{(j)}$ of the oscillator $(i, j)$ at AEP and PEP are determined as follows;

$$
\hat{\phi}_{i}^{(j)}=\hat{\phi}_{i A}^{(j)} \text { at AEP, } \quad \hat{\phi}_{i}^{(j)}=0 \text { at PEP }
$$




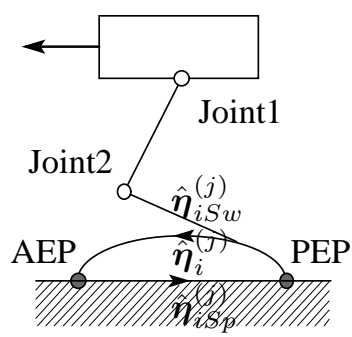

Figure 3: Nominal trajectory of the leg

We use one of these two trajectories alternatively to generate the nominal trajectory $\hat{\eta}_{i}^{(j)}$ of the tip of the leg as follows(Fig.3);

$\hat{\eta}_{i}^{(j)}\left(\hat{\phi}_{i}^{(j)}\right)= \begin{cases}\hat{\eta}_{i S w}^{(j)}\left(\hat{\phi}_{i}^{(j)}\right) & 0 \leq \hat{\phi}_{i}^{(j)}<\hat{\phi}_{i A}^{(j)} \\ \hat{\eta}_{i S p}^{(j)}\left(\hat{\phi}_{i}^{(j)}\right) & \hat{\phi}_{i A}^{(j)} \leq \hat{\phi}_{i}^{(j)}<2 \pi\end{cases}$

The nominal duty ratio $\hat{\beta}_{i}^{(j)}$ of leg $j$ of module $i$ is the ratio between the nominal time for the supporting stage and the period of one step of the nominal locomotion, which is given by

$$
\hat{\beta}_{i}^{(j)}=1-\frac{\hat{\phi}_{i A}^{(j)}}{2 \pi}
$$

Then, the nominal stride $\hat{S}_{i}^{(j)}$ of leg $j$ of module $i$ and the nominal locomotion velocity $\hat{v}$ are given as follows;

$$
\hat{S}_{i}^{(j)}=\hat{\eta}_{i A 1}^{(j)}-\hat{\eta}_{i P 1}^{(j)}, \hat{v}=\frac{1-\hat{\beta}_{i}^{(j)}}{\hat{\beta}_{i}^{(j)}} \frac{\hat{S}_{i}^{(j)}}{\hat{T}_{S w}}
$$

where $\hat{T}_{S w}$ is the nominal time of the swinging stage.

The nominal angle $\hat{\theta}_{i k}^{(j)}$ of joint $k$ of leg $j$ of module $i$ is written as a function of nominal phase $\hat{\phi}_{i}^{(j)}$ as follows;

$$
\hat{\theta}_{i k}^{(j)}=\hat{\theta}_{i k}^{(j)}\left(\hat{\phi}_{i}^{(j)}\right)
$$

The commanded torques at each joint of the legs are designed using the nominal angle $\hat{\theta}_{i k}^{(j)}$ as the commanded angle as follows;

$$
\tau_{i k}^{(j)}=K_{P i k}^{(j)}\left(\hat{\theta}_{i k}^{(j)}-\theta_{i k}^{(j)}\right)+K_{D i k}^{(j)}\left(\dot{\hat{\theta}}_{i k}^{(j)}-\dot{\theta}_{i k}^{(j)}\right)
$$

where $\tau_{i k}^{(j)}$ is the actuator torque at joint $k$ of leg $j$ of module $i$, and $K_{P i k}^{(j)}, K_{D i k}^{(j)}$ are the feedback gains.

On the other hand, the commanded torques at each joint of the couplers are obtained as follows;

$$
\tau_{i}=-K_{P i} \theta_{i}-K_{D i} \dot{\theta}_{i}
$$

where $\tau_{i}$ is the actuator torque at coupler $i$, and $K_{P i}, K_{D i}$ are the feedback gains.

\section{Straight walk}

Here, we analyze the stability of the straight walk of the multi-legged locomotion robot with the control system of locomotion described above.

\subsection{2-D dynamics model}

First, it is assumed that the robot walks with a gait pattern where many of the legs always make contact with the ground. Then, the vertical motion of the robot can be neglected. The robot snakes its way on the ground under the influence of the forces acting from the ground intermittently. Next, it is assumed that the period of the meandering motion is long as compared with that of the cyclic motions of the legs. Then, the intermittent forces from the ground may be modeled as the constant forces and the speed of locomotion of the robot may be constant.

From these assumptions, the 3-D model shown in Fig. 1 are simplified by the 2-D model shown in Fig.4. This model is composed of the rigid bodies which are connected to each other through a one degree of freedom rotational joint, and can move only in a plane. As propulsive forces, the constant follower forces parallel to the walking directions of each modules act on the modules. As friction forces, the forces tangential to the walking directions of each modules act on the modules and the magnitudes of the forces are proportional to the velocities of these directions.

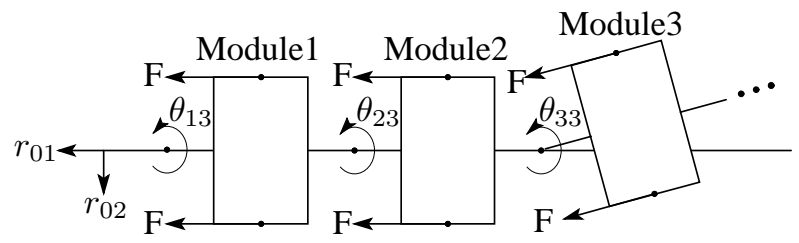

Figure 4: Simplified model of multi-legged locomotion robot

From these assumptions, Eq.(2) is simplified as follows;

$$
\begin{gathered}
{\left[\begin{array}{cc}
M(q) & 0 \\
0 & I
\end{array}\right]\left[\begin{array}{c}
\ddot{q} \\
\dot{q}
\end{array}\right]=\left[\begin{array}{c}
f(q, \dot{q}) \\
\dot{q}
\end{array}\right]} \\
q^{T}=\left[r_{02}, \theta_{i 3}\right](i=1, \cdots, 6)
\end{gathered}
$$

where the state variable of the walking direction, $r_{01}$, is contracted because it is separated from the other equations. By putting $\xi^{T}=\left[\dot{q}^{T}, q^{T}\right]$ Eq.(14) becomes as follows;

$$
\begin{gathered}
A(\xi) \dot{\xi}=b(\xi) \\
A(\xi)=\left[\begin{array}{cc}
M(\xi) & 0 \\
0 & I
\end{array}\right], b(\xi)=\left[\begin{array}{c}
f(\xi) \\
B \xi
\end{array}\right], B=\left[\begin{array}{ll}
I & 0
\end{array}\right]
\end{gathered}
$$




\subsection{Linearized equation and its stability}

We define $\xi_{0}$ as the equilibrium solution of Eq.(15). We expand Eq.(15) about the point $\xi_{0}$ as follows;

$\left\{A\left(\xi_{0}\right)+\left.\frac{\partial A(\xi)}{\partial \xi}\right|_{\xi_{0}} \delta \xi\right\} \dot{\delta \xi}=b\left(\xi_{0}\right)+\left.\frac{\partial b(\xi)}{\partial \xi}\right|_{\xi_{0}} \delta \xi(16)$

where $\xi=\xi_{0}+\delta \xi$. It is linearized as follows;

$$
A\left(\xi_{0}\right) \dot{\delta \xi}=\left.\frac{\partial b(\xi)}{\partial \xi}\right|_{\xi_{0}} \delta \xi
$$

Since $A\left(\xi_{0}\right)$ is regular, Eq.(17) is rewritten as

$$
\dot{\delta \xi}=W\left(\xi_{0}\right) \delta \xi, \quad W\left(\xi_{0}\right)=\left.A\left(\xi_{0}\right)^{-1} \frac{\partial b(\xi)}{\partial \xi}\right|_{\xi_{0}}
$$

We select $\xi_{0}=\left[\dot{y}, \dot{\theta}_{i}, y, \theta_{i}\right]=[0, \cdots, 0](i=$ $1, \cdots, 6)$ as the equilibrium solution in order to analyze the stability of the straight walk. Parameters are shown in Table 1.

Table 1: Linear analysis parameters

\begin{tabular}{|c|c|c|}
\hline Mass of Module & 0.80 & {$[\mathrm{~kg}]$} \\
\hline Follower Force $(F)$ & 3.32 & {$[\mathrm{~N}]$} \\
Locomotion Velocity $(\dot{x})$ & 0.167 & {$[\mathrm{~m} / \mathrm{s}]$} \\
\hline
\end{tabular}

First, we investigate the stability of the straight walk when the compliances of yaw joints of the couplers are increased. The feedback gains is changing by using the parameter $f$ where parameter $\zeta$ is set to be 0.8 .

$$
\left\{\begin{array}{l}
K_{P i}=K_{0}(2 \pi f)^{2} \\
K_{D i}=2 K_{0} \zeta(2 \pi f)
\end{array} \quad(i=2, \cdots, 6)\right.
$$

The trajectories of the eigenvalues of the linearized Eq.(18) is shown in Fig.5. From this figure, it is revealed that when the parameter $f$ decreases and reaches a critical point, a pair of eigenvalues cross the imaginary axis and become unstable. That is, a Hopf bifurcation occurs. In the figure, the points enclosed by circles correspond to the eigenvalues when the bifurcation occurs. From this analysis, it is revealed that when the feedback gains of yaw joints of the couplers are big, the straight walk of the robot are stable, but when they are small beyond a bifurcation point, the straight walk becomes unstable.

\subsection{Numerical analysis}

We verify the liner analysis of 2-D model by the numerical simulations.

Parameters are shown in Table 2. The nominal gait pattern of the robot is set so that the leg 1 and 2 of each

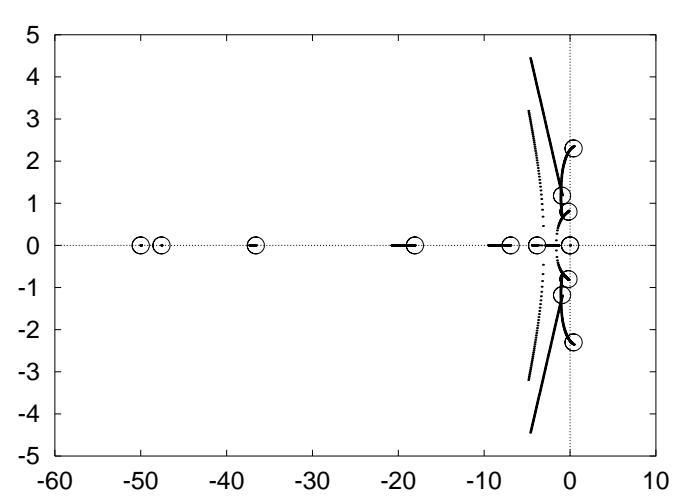

Figure 5: Trajectories of eigenvalues

Table 2: Simulation parameters

\begin{tabular}{|c|c|c|}
\hline Mass of Body & 0.60 & {$[\mathrm{~kg}]$} \\
\hline Length of Link 1,2 & 0.07 & {$[\mathrm{~m}]$} \\
Mass of Link 1,2 & 0.050 & {$[\mathrm{~kg}]$} \\
\hline Nominal Time of the & 0.2 & {$[\mathrm{~s}]$} \\
Swinging Stage $\left(\hat{T}_{S w}\right)$ & & \\
Nominal Stride $\left(\hat{S}_{i}^{(j)}\right)$ & 0.05 & {$[\mathrm{~m}]$} \\
\hline
\end{tabular}

modules and the unilateral legs on adjacent modules move out of phase each other.

First, we investigate the behavior of the robot when the compliances of yaw joints of the couplers are increased. The feedback gains is changed by the parameter $f$. The nominal duty ratio $\hat{\beta}_{i}^{(j)}$ is set to be 0.6. The results are shown in Fig.6. This figure shows the Poincaré sections of the relative angle $\theta_{43}$ of yaw joint of coupler 4 against the parameter $1 / f^{2}$. The sampling time is a period of motion of the legs. This figure indicates that there is a critical point and beyond that the robot begins to snake its way. In this figure, vertical lines of the each Poincare sections are filled because motions of the legs and the meandering of the robot do not synchronize. This bifurcation does not depend on the gait patterns of the robot; There exists a similar bifurcation if the gait pattern of the robot is the pattern where the leg 1 and 2 of each modules move in phase and the legs on adjacent modules move out of phase. Moreover, it is verified that the period of the meandering motion of the robot is much longer, about 14 times longer, than that of the leg movements.

Next, we compare the values of state variables of the meandering motion obtained from numerical simulations with the unstable eigenvectors of the linear analysis. Figures 7 and 8 show the amplitudes of the joint angles and the phase differences of the joints with the joint of module 6 against the parameter $1 / f^{2}$. These figures indicate that the results of the numerical simulations and the linear analyses resemble especially near 


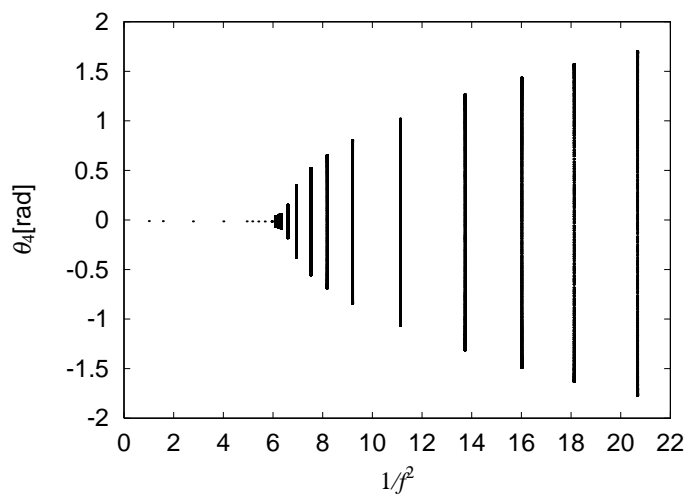

Figure 6: Poincaré section of $\theta_{43}$

the bifurcation point (arrow).

\section{Turning walk}

\subsection{Turning strategy}

Based on the results described above, we propose a turning strategy as follows;

1. When the robot start to turn, the compliances of all coupler except for coupler 2 are increased, that is, the gains of yaw joints of the couplers are decreased.

2. The control torques of yaw joints of coupler 2 are activated so as to make first module face the direction to turn.

In order to turn the robot, this control system is to make first module face the direction to turn, and as a result the modules follow first module because they are passively connected to each other. It may be noted that, at that time, the modules can turn with less constraint forces because the robot has less stability of the straight walk.

\subsection{Numerical analysis}

We verify the effectiveness of the proposed control strategy by the numerical simulations. A task is set that the robot goes to a destination point. We assume that the robot can get the information about the relation between the destination point $\left(x_{d}, y_{d}\right)$ in $\left[\boldsymbol{a}_{1}\right]$ system. The direction angle $\psi$ for first module to turn is given as follows.

$$
\psi=\frac{\pi}{2}-\phi, \quad \tan \phi=\frac{x_{d}}{y_{d}}
$$

The control torque of yaw joint of coupler 2 is given as

$$
\tau_{23}=-K_{P 23}\left\{\theta_{23}-\left(\theta_{13}-\psi\right)\right\}-K_{D 23} \dot{\theta}_{23}
$$

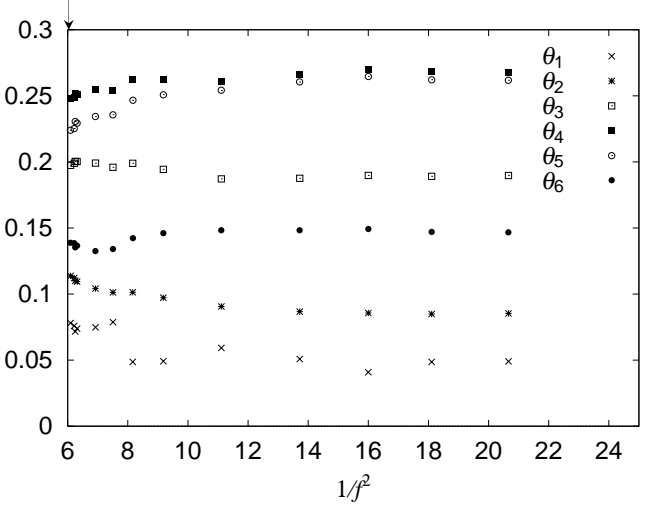

(a). Numerical simulation

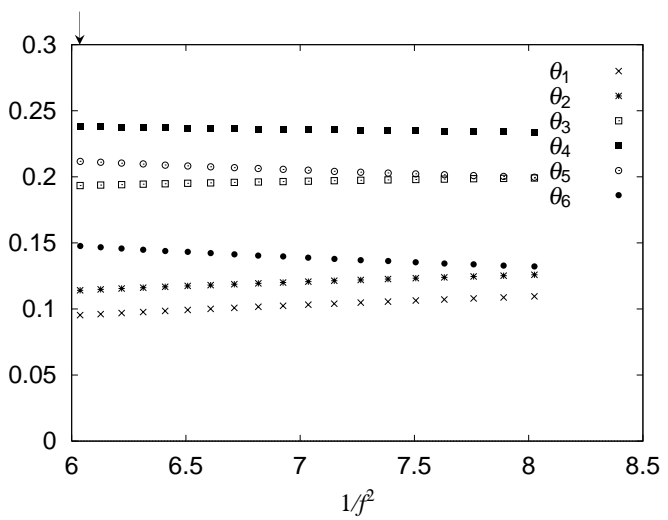

(b). Linear analysis

Figure 7: Amplitudes of joint angles

At that time, the compliances of yaw joints of the other couplers are increased whereas the compliance of yaw joint of coupler 2 is set to be constant. Numerical simulations carried out as follows; when the robot walks toward the direction of $x$-axis and first module reaches the point $(x, y)=(0,0)[\mathrm{m}]$, the robot is to turn almost perpendicularly by setting the destination point $(x, y)=(0,50)[\mathrm{m}]$.

Fig.9 shows the change of the direction of first module for three kind of the feedback gains. Fig.10 shows the average slippage of each legs to the ground against the parameter $f$.

From these results, it is revealed that in the region where the compliances are so small, that is, the feedback gains are so big, the modules can not follow first module efficiently, slippage of the legs is so big, and many modules are lugged. On the other hand, in the region where the compliances are so big, that is the feedback gains are so small, the modules can follow efficiently but because the meandering motion occurs, the legs slip and the robot can not walk efficiently. When the feedback gains are set to be just before the gains in which the straight walk of the robot begins to be unstable the robot can walk most efficiently. 


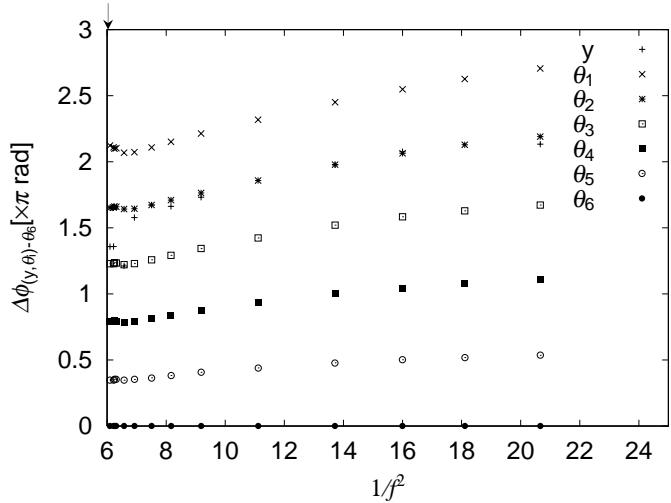

(a). Numerical simulation

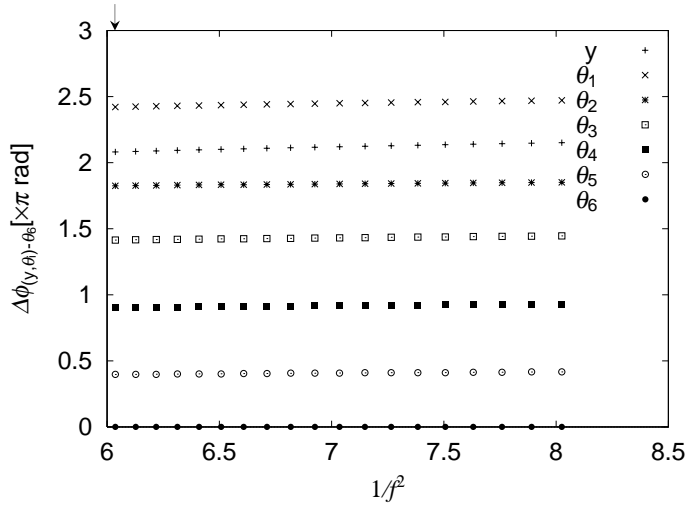

(b). Linear analysis

Figure 8: Phase differences of joints with the joint of module 6

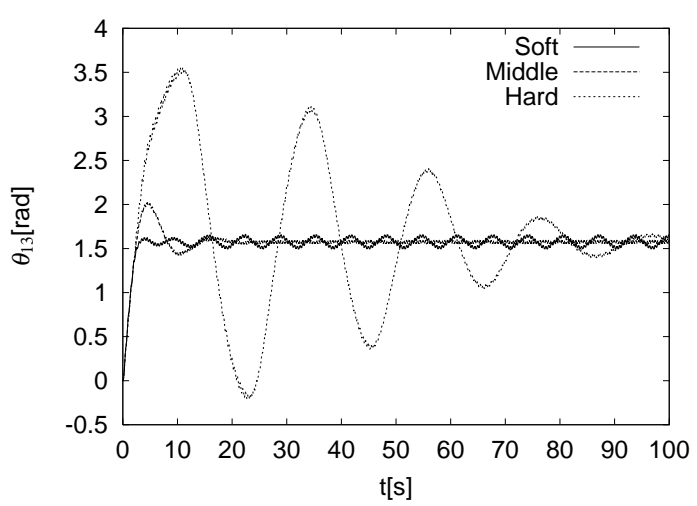

Figure 9: Walking direction of module 1 to inertial space

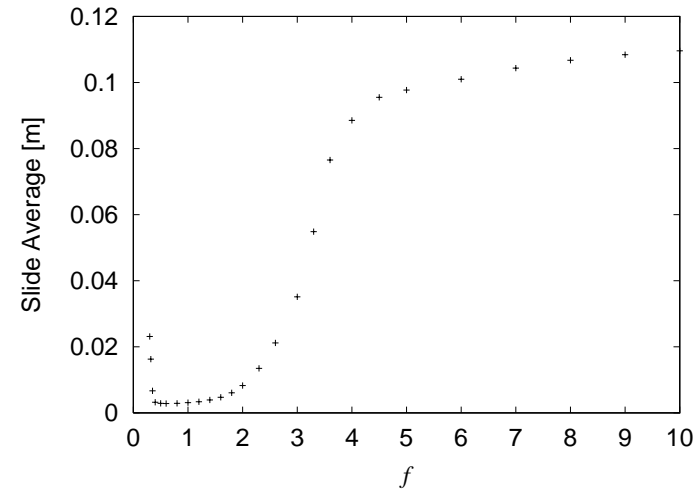

Figure 10: Average slippage of each legs

It is concluded that the robot can turn with less slip to the ground by decreasing the stability of the straight walk of the robot. It should be noted that this means that the maneuverability is increased by the compliance controls.

\section{Acknowledgments}

The authors were funded by grants from the Japan Science and Technology Corporation (JST) as the Core Research for Evolutional Science and Technology Program (CREST).

\section{References}

[1] Jindrich D, Full RJ, 1999, "Many-legged maneuverability: dynamics of turning in hexapods," J Exp Biol 202, pp. 1603-1623.

[2] Schmitt J, Holmes P, 2000, "Mechanical models for insect locomotion: dynamics and stability in the horizontal plane - application," Biol Cybern 83, pp. 517-527.

[3] Kazuo Tsuchiya, Katsuyoshi Tsujita, Manabu Kawakami, Shinya Aoi, 2001, "An emergent control of gait patterns of legged locomotion robots, " IAV2001, pp. 271-276. 